\title{
A framework to study learning in a complex learning environment
}

\author{
Anders Berglund* \\ Uppsala University
}

This paper presents a framework for analysing variations in the ways in which students experience learning in complex, computer-supported environments. It involves an application of phenomenography extended to encompass the content of the learning as well as its context. Concepts from activity theory are drawn upon to enlarge the scope of phenomenography. By applying this framework, researchers, as well as course organisers, may gain insights into how courses are experienced by the participants and can thus develop these in a way that will be perceived as improvement by the course participants. Within this predominantly phenomenographic approach, the focus is upon the content of the learning experience, giving the ability to relate learning outcomes to the experience of the learning environment. Hence, insights can be gained that can help to improve learning environments and thereby outcomes. A case study illustrating the kinds of results than can be obtained when using the framework is provided.

\section{Introduction}

Within this article a research framework is proposed that is intended to support the study of the complex interplay between learning in a subject area and the learning environment. The development of this framework is based on the needs of a research project in which computer network concepts are studied in an internationally distributed project-based course. In the course, the students work in virtual teams, collaborating using different learning technologies. The aim of the framework is to provide a tool that researchers can use to analyse and describe learning within a subject area, taking account of the complex learning environment in which this takes place and leading to insights into how the students experience this learning environment. To achieve this goal, it is crucial to understand the role of the learning environment from the students' perspective, since changes in a course that are not related to students perceptions run the risk of being useless or even counter-productive.

\footnotetext{
*Department of Information Technology, Uppsala University, PO Box 325, SE-75105 Uppsala,
} Sweden. Email: Anders.Berglund@it.uu.se 
The focus on the learner's experience suggests the use of a phenomenographic research approach (Marton \& Booth, 1997), since phenomenography aims at revealing learners' relationship with, or experience of, different phenomena in the learning environment and in the subject area. The different ways in which phenomena under investigation are experienced (or understood) are highlighted in a phenomenographic research project, while other issues are relegated to the background. In this way, the researcher gains insights into the different constituents that, in their interplay, create the experience of learning in a particular environment; however, they risk loosing sight of the whole picture. The danger is that the researcher may gain a fragmented view in which the constituents of the environment are seen in isolation. To address this issue, the framework proposed here uses concepts drawn from activity theory (Engeström, 1987) to study the phenomenographic outcomes in greater detail. Activity theory serves as a vehicle for the researcher to integrate the outcome of phenomenographic analyses to provide a more holistic account. Importantly, if activity theory were to be used without reference to the phenomenographic results, the learner's perspective would be lost, and, as consequence, the aims of the research could not be addressed. It is thus a combination of these two approaches, where phenomenography is extended with elements of activity theory, that is advocated here.

The remainder of this article is structured as follows. Phenomenography, which provides the theoretical basis for this project, is described in the next section, and is followed by a discussion of some elements of activity theory. I then present the research framework and a case study that is used to illustrate its application. Finally, some implications of the framework are discussed.

\section{An introduction to phenomenography and activity theory}

\section{Phenomenography}

Phenomenography offers the possibility of investigating issues related to students' varying experiences of their learning and their situations. With its object of study being the relationship between a learner and a phenomenon, the outcome becomes a description of this relationship as a limited set of qualitatively different ways in which the phenomena are experienced, or understood, by some learners. Variation is in focus in phenomenography; individuals are understood as being 'carriers' of different ways of experiencing something or else of fragments of a collective way of experiencing it. The results are then interpreted at a collective level, without relating a particular way of understanding something to a specific individual or to groups of individuals.

With this as a point of departure, individual learning is interpreted, or defined, as acquiring the capacity to see something in a qualitatively new way. Learning, understood in this way, is shaped both by the learners and the phenomena they study. The role of the researcher is to analyse and describe this relationship, that is, to reveal the variation in the ways in which the students experience phenomena. Both the students and the concepts that they study are present in the outcome, which is a description of 
the researcher's understanding of the various ways in which the students understand some concepts.

A strong focus on the content of the subject area, giving rise to the possibility of influencing education within that field, has been promoted by several authors as a key feature of phenomenography (for example: Berglund, 2002a, in computer networks; Booth, 1992, in programming; Cope, 2000, in information systems; Pang, 2003, in business economy). The discussion in Berglund (2002a), concerning students' learning of a particular network protocol, can serve as an illustration. In this paper I argue that a teacher who understands the different ways in which students perceive the phenomenon being taught can use these insights as a tool to improve her teaching.

In the phenomenographic tradition, issues related to the learning environment and to its role in learning are normally relegated to the background, since "the analysis deliberately strips away contextual features of the data in order to focus clearly and exclusively on the phenomenon" (Adawi et al., 2001, p. 82). For the current project, where learning within the subject area is studied both within, and as a part of, a particular learning environment, the use of phenomenography alone as a guide for the research is therefore not sufficient. For this reason, I propose a phenomenographic approach that is informed by elements of activity theory as a unifying framework for this kind of research.

\section{Activity theory}

The elements of activity theory, as they are employed in this project, serve to describe, analyse and explain human activities, such as learning, as integrated parts of an environment. The theoretical aspects of activity theory are based on the interpretations of Engeström (1987) as well as other researchers in this tradition (Bannon, 1997; Bellamy, 1996; Kaptelinin et al., 1995; Kuutti, 1996; Nardi, 1996). Activity theory is briefly presented here and is further described by Berglund (2002b), with a focus on the needs of this phenomenographic research project.

With an activity theoretical approach, learning can be understood as being integrated in a larger system that considers the socially based nature of human activity. Activity theory serves here as a "clarifying and descriptive tool rather than a strongly predictive theory" (Nardi, 1996, p. 7).

An activity system describes the interaction between a subject (in this study, a student), and an object (a programming task, or a understanding a computer science concept). The activity, as a whole, is directed towards this object, which is transformed into an outcome. This transformation of the object is the reason for the existence of the activity. The subject interacts with the object through tools, both physical (such as a computer) and intellectual (abstract computer science concepts). The activity is, however, larger than the tool-mediated relation between a subject and an object. It is an integrated part of a collective activity involving a community that shares the same object (other students taking the same course, university staff). The relationship between the subject and the community are constrained by rules. The term 


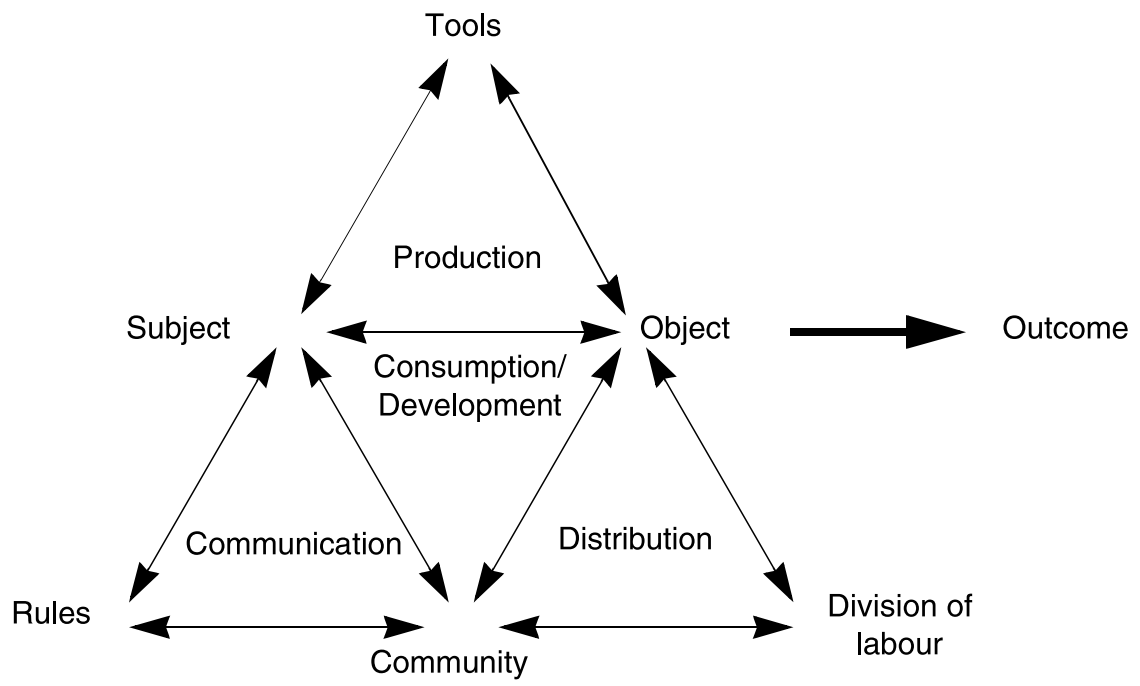

Figure 1. An activity system (developed from Engeström, 1987, p. 78)

'rules' must be understood in a broad sense, including aspects as diverse as the legal systems, cultural or social rules for people's interaction as well as the rules, or habits that have developed within the current activity. The relationship between the community and the object itself is described by the division of labour.

The components and their relationships are frequently presented graphically, as shown in Figure 1. The sub-triangles describe different aspects of the broader activity and can in themselves often be analysed as activity systems (Engeström, 1987). The sub-triangle in the top would then describe production, while the lower left sub-triangle describes exchange (or communication). The lower right triangle expresses the distribution activity. Finally, the middle triangle (which in Engeström's model, following a Marxist tradition, is labelled consumption) is here denoted development.

An activity is continuously constructed and reconstructed by the individuals within the system. In this way, the activity becomes more than the sum of individual discrete actions, and the individual becomes inseparably intertwined with the activity.

As the object is what motivates the existence of the activity, the activity in its turn gives "meaning to seemingly random individual events" (Engeström, 1993, p. 65). That is, an activity is a context in which the participating individuals and the different events are integrated parts. With this approach, it is impossible to study a part of the activity separated from the whole system. The activity as a whole becomes the unit of analysis. This does not imply that the activity is a homogenous and harmonious entity; on the contrary, with its multi-voiced character and its embedded history, it contains different, often contradictory, expressions and events that serve as a ground for inner conflicts. These conflicts, in their turn, are important forces in the development of the activity. 


\section{Using the two approaches together}

An extensive literature survey has indicated that only a few research projects use phenomenography and activity theory within the same project. Significant work in this context includes that of Åberg-Bengtsson (1998) concerning children learning graphs, Gordon (1998) concerning students' learning of statistics, Coupland and Crawford (2002) concerning computer-based tools in learning mathematics and Hultén and Booth (2002) concerning learning within a collaborative project-based distance course.

These studies all combining the research approaches in different ways. However, they use phenomenography for aspects that are close to, or perceived by, the learners, while the role of activity theory is to analyse and/or describe a context for this learning. Hultén and Booth takes a stand similar to the one presented here: they adopt a phenomenographic approach illuminated by elements of activity theory, while ÅbergBengtsson, as well as Gordon, choose to let the two approaches serve as different lenses that illuminate separate aspects of learning. Coupland and Crawford, finally, propose a predominantly activity theoretical approach, where results from methods inspired by phenomenography offer data for the activity theoretical analysis.

The focus in activity theory on a particular situation, seen as a whole, implies that the actions taken or performed by individuals within the activity form an important basis for the data used in the analyses. In a phenomenographic study, on the other hand, which aims to reveal the different ways in which something is experienced, the learners' own accounts play important roles. This discrepancy is clearly voiced by Ekeblad and Bond (1994) in terms of a difference between an externalist perspective, which leads researchers to observe events and to analyze them from within their theoretical and methodological frameworks, and an experiential perspective, in which the researcher seeks to see events as the actors experience them.

The research framework presented here serves as a tool to study the experience of learning in the perceived context by applying an experiential perspective on the students' learning environment. Hence the phenomenographic foundations are kept in the proposed research framework, even when studying the context of the learning, and the potential conflicts between the approaches are avoided.

\section{Extending the use of phenomenography}

Marton and Booth (1997, p. 113) define the research object of phenomenographic research in the following terms:

The unit of phenomenographic research - a way of experiencing something- $[\ldots]$ is an internal relationship between the experiencer and the experienced.

Taking this as the point of departure for the research framework, the issue of relating it to, or extending it with, the experienced context becomes crucial.

An activity is, as described earlier, a theoretical entity, discerned and described by the researcher who aims to study the context within which the subjects (in this study: students) and the object (what is learnt) are integrated parts. Someone, a subject, 
cannot experience the complete whole at a particular point of time, since, as Marton and Booth point out, different parts or aspects of the world are within the focus of the attention at different times. A student can, for example, focus at a particular moment on some aspect of a concept within the course content, such as a networking protocol, whilst another issue, such as concerns about decision-taking in the team, are relegated to the background. Later, focus might shift to decisions concerning the distribution of work, with the concepts of network protocols residing in the background. The students' relationships to these phenomena within the environment are thus dynamically changing.

Both learning the course content (here network protocols) and experiencing other phenomena related to the course (for example, a team structure) are important for a researcher to describe and analyse if he/she are to understand the students' experience of their studies in a particular setting. Both are needed to understand the complex relationship between the learning outcome and the experience of the learning environment.

In the framework presented here, the students' experience of these phenomena, whether directly related to the learning objectives or not, are analysed in the phenomenographic tradition. The outcome of a phenomenographic analysis is sets of categories of description, where each set describes the experiences of a particular phenomenon found within a team, and each category describes a certain way in which the phenomenon is understood.

These categories and their relationships are then further analysed based on elements drawn from activity theory. The outcome of the 'pure' phenomenographic analysis is thus not the final outcome of the research project. In addition to its role as an outcome in itself, this phenomenographic account serves as a material for analysing the learning in a particular situation as it is perceived or experienced by its participants. The final outcome of the analysis consists thus of a description of the students' experience of learning of the subject area within a particular course setting, as it is experienced.

\section{Methodology: a phenomenographic analysis of an activity}

As briefly indicated above, the analysis is performed in two steps. First, variations in the students' experience of different phenomena in the learning environment are analysed and described, following the phenomenographic tradition. As a second step the analysis is advanced by means of some intellectual tools related to activity theory. Here the outcome space of the phenomenographic analysis is associated with different components of an activity system. In this way, the researcher gain means to further explore his/her phenomenographic results by deploying concepts from activity theory. The aim is to create the potential to capture, analyse and describe the variation in the participants' experience of the whole.

As a starting point for this analysis, each category of description is associated by the researcher with one or more component(s) of an activity system. This association demands of the researcher that he/she 'goes inside' the activity and explores it from 
the participants' perspective. Through such associations, each component of the activity comes to have some phenomenographic categories related to it that together indicate the variation in how this particular component is experienced by the subjects. As will be demonstrated in the case study, the various categories related to a particular phenomenon can be associated to different components, and the categories at each component can, as a consequence, contain descriptions of various phenomena.

The activity system, now describing the various ways in which the learning situation is experienced by its participants, is further analysed by deploying some intellectual tools of activity theory. To structure such an analysis, it is useful to base the analysis on the three different levels of the concept of context within phenomenographic research, which we introduce in Adawi et al (2002):

1. The experienced context of the individual describes the relationship between a phenomenon and its context, and is discussed as follows. During a "phenomenographic interview, some aspects of the phenomenon come into focus, while others remain in the background. The phenomenon is thus experienced against and interwoven with an experienced context, what we can refer to as the experienced context of the individual" (italics in original).

2. The experienced context of the collective describes the interplay between utterances of different individuals. "When analysing the interviews, the researcher finds that light is shed on some utterance made by one interviewee by reading it against the background of the context deduced or assumed by the researcher from reading an interview extract by another interviewee. Switching between these two perspectives allows the researcher to let an aspect of a phenomenon as experienced by one participant interplay with an expression of an experienced context that originates from another participant".

3. The experienced context of the researcher describes the researcher's relationship to the object of his/her research: "it can be claimed that when engaged in a phenomenographic study on learning the researcher stands in the same relationship to the object of research as the learner stands to the object of learning. The object of research is embedded in a context, and this context can be said to be what lends meaning to the object".

These distinctions, which are based on an analysis of who the experiencer is, are here extended to encompass the concepts of an activity system. The phenomenographic outcome space, now related to the components of an activity system, can be analysed in the following ways:

1. At the individual level, case studies are made where individuals are studied in the light of the activity. Relations and tensions in the different ways in which an individual experiences a learning environment give the researcher a 'feel' for the individual in the activity.

2. At the collective level, the inner tensions are described and analysed. As stated earlier, the tensions within a system are its source of development and change. Analysed in this way, the tensions between and within the experienced constituents 
are discriminated and discerned, offering a way to understand the dynamics of the learning processes in the team.

3. At the researcher's level, a further phenomenographic analysis can be performed by returning to the interview excerpts that now, through the phenomenographic categories, are related to a particular component. These interview excerpts can be analysed again, using the component and the earlier analyses as a background. The new analyses can be structured to reveal aspects related to the component of the activity in question and to those phenomena that originally, through the different categories, fed into the description of the component. By performing this analysis, the researcher can gain insights into the learners' experience of a particular component of the activity.

Analyses at these three levels, taken together, offer to researchers and course organisers tools that can be used for understanding learning in real situations as experienced by the participants.

\section{Bringing the research framework into play: a case study}

An example from the on-going project will serve as a case study and will illustrate how the framework can be used by a researcher as a vehicle to draw conclusions about the students' experience of their learning environment. The results presented here are not the final result of the research project. Instead, the case study shows the framework 'in action' and thus offers insights in the methodology rather than the outcome.

In the course that serves as the core for this research project advanced students, majoring in computer science, work in teams of six. Each team consists of three members from a Swedish university (Uppsala University) and three from the USA (Grand Valley State University) and was assigned the task to jointly produce a software package intended to control a hardware setup, in the form of a motorized and computerized toy, from any web-browser. (See Figure 2, from Berglund, 2002a; The aim of the game is to move a steel ball on the board from a starting point to a final point. The knobs are used to tilt the board and in this way move the ball.) The task is considered to be hard and demanding both by students and teachers. To reach a solution the team has to work as a unit, using different Internet-based tools for their communication (Daniels, 1999).
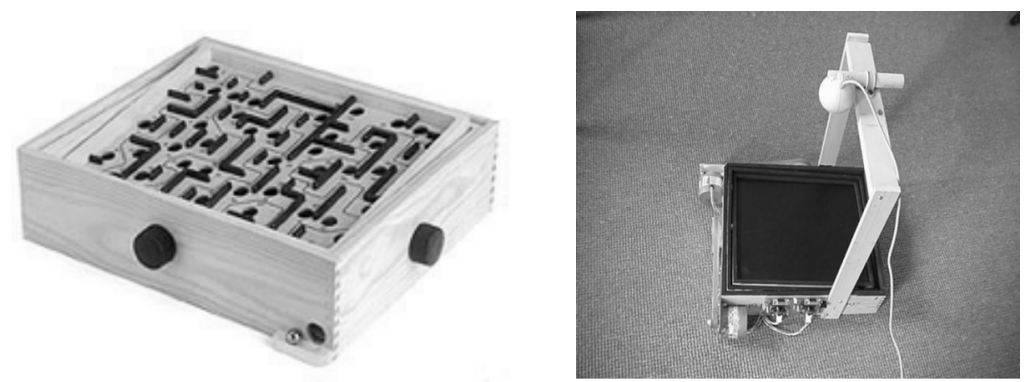

Figure 2. A Brio labyrinth and a modified version with a camera and motors added 
The results presented here are based on interviews with 15 students: 8 in Sweden and 7 in the USA. They were selected to represent diversity in background factors such as age, previous educational background, earlier study results within computer science and factors obtained from a background questionnaire (expectations about the project work and their own contribution to the group work, motivation to take this particular course and to study computer science in general). The aim of the selection was to attain a rich phenomenographic outcome space, where different voices about the phenomena under investigation were heard and from which the researcher could discern and described a nuanced picture of relevant experiences within a cohort. The students were interviewed at two occasions about their understanding of computer science concepts relevant to the project as well as their experience of taking this particular course in its internationally distributed setting (Berglund, 2002a).

\section{The experience of being a team member}

Membership in a team is one of the more important factors constituting a student's experience of his/her learning environment. What it means to be a member of a particular team is settled in a continuous interplay between an individual and his/her team. Such relationships are dynamic: the relationships between the actors, as well as the actors themselves, change continuously. An example can illustrate this interplay: a student who acts (for example by arguing that a particular task should be done as the next step) changes not only himself (by taking a stand) but also the team (for example, by making the team focus on something new). The relationship between the student and the team (of which he/she is a part) also changes with his/her argument: he/she might, for example, get a more central position as a result of the discussion. The complex environment that the students face in this project, where the complete team never meet face to face, brings these matters to a head.

The on-going project has explored these issues from a phenomenographic perspective, and has revealed two factors that together play an important role in shaping a student's experience of being a team member. These are (1) the experience of the parts, of which the team is constituted, and (2) the relationships between these parts, experienced as a control structure within a team. While the first describes the structure of the team that a student experiences, and addresses the issue of the parts or units that constitute the team, the latter highlights the capacity to take and implement decisions within a team. Here a complex picture evolves that expresses itself in issues such as the roles of the leader and the team members, as well as in their relationships. Aspects concerning the organisation (or lack of organisation) of a team also become visible.

Four qualitatively different ways of experiencing the control structure were discerned, described elsewhere in greater detail (Berglund, in press):

1. Control is distributed. Here individuals are active participants in the control structure. Decisions are jointly taken, with the role of the team leader being to coordinate and facilitate communication.

2. Control is allocated. The capacity to take decisions lies with the team members but is allocated to a team leader. The team leader distributes tasks to the participants and offers support in a working two-way communication with the team members. 
3. Control is taken by a few. A sub-team (or an individual) takes control of the activities in the team, where they (or he/she) enforce the decisions. Control is unevenly distributed, with one-way communication taking place from the centre of control to more peripheral team members.

4. Control is missing. In the absence of a defined, stable structure, different ad hoc subgroups try to gain control in an ongoing social game. The situation of an individual team member varies with the changes in the control structure.

The first two categories will be further discussed and contrasted in this case study. The analysis here aims to illuminate and explore the phenomenographic results and is performed according to the framework presented in the previous sections. This example will demonstrate how, through the use of concepts of activity theory, deeper insights are gained into the phenomenographic findings.

The first category depicts a situation where control is shared. The forms for the communication in the team are negotiated (communication sub-triangle, lower left corner in Figure 3), which is constituted by a subject (a team member), the community (the full team of six) and the rules, norms and traditions that evolve in the discussion or that are brought into the situation. Here, the relationship between an individual and the team crystallises. At the same time, and as a part of the same process, a structure for how decisions are taken is created. The sub-triangle in the lower right corner of the same figure (here labelled coordination) depicts how the object (the software package that the team work upon) is distributed over and coordinated between the team members in an on-going process. Finally, the development (or consumption) triangle characterizes the subject's striving to transform the object, an effort that takes place within and with the assistance of the community.

A particular feature of this category is indicated in the strong position that is held by the team, both in the creation of rules and in the distribution of control and labour. The value of an open communication that offers possibilities to renegotiate the rules has been reported during the interviews. The control of the object is experienced as jointly shared, orchestrated by a team leader with a coordinating function. This implies that the subject experiences himself to be a part of the control structure, as illustrated by arrow $a$ in Figure 3. The picture of the experience of the control structure, as drawn here, suggests both through the absence of visible conflicts and by its capacity to cope with changes that the category under investigation is harmonious and functional.

A similar picture can be discerned for the second phenomenographic category, but of course, there are differences, as illustrated in Figure 4. The most significant discrepancies, compared with the previously discussed category, lie in the different meanings of the components of the activity system. Differences are found both in the role of the subject (which here offers parts of his/her capabilities to a selected team leader) and the team (which here, as a unit, becomes less involved in the on-going control). Arrow $a$ in Figure 3 becomes less important and would now describe the experience of two-way communication, instead of experienced participation. For this reason it is omitted in Figure 4. To further emphasize the changes, the triangle to the right is re-labelled to distribution. 


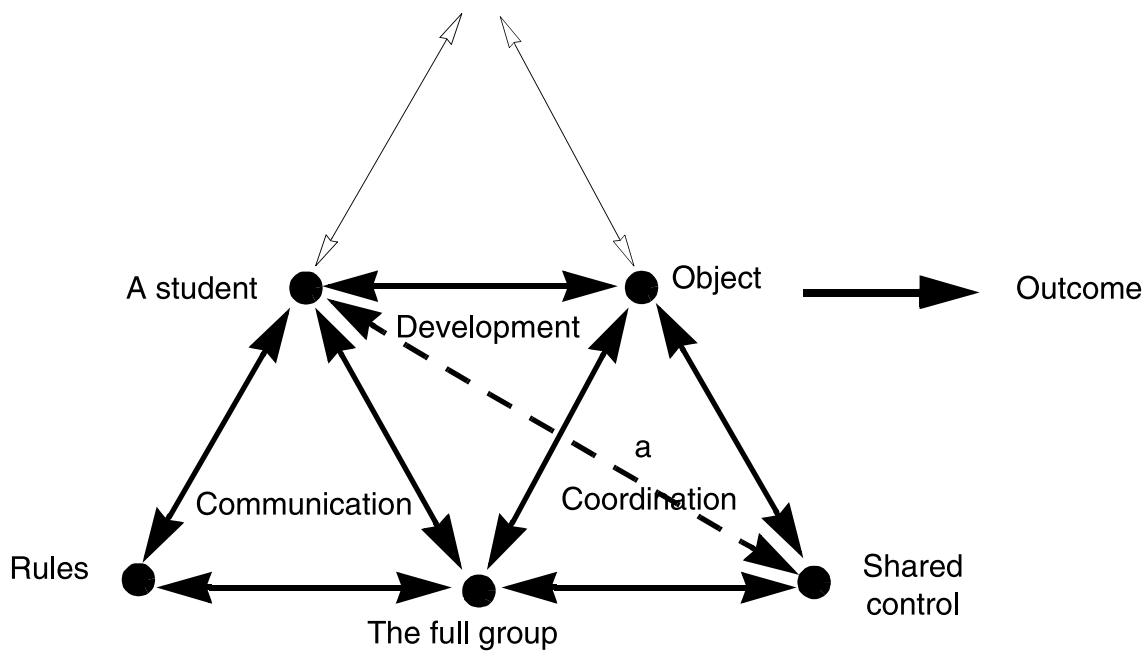

Figure 3. Relating the category Control is distributed of the experienced control structure to an activity
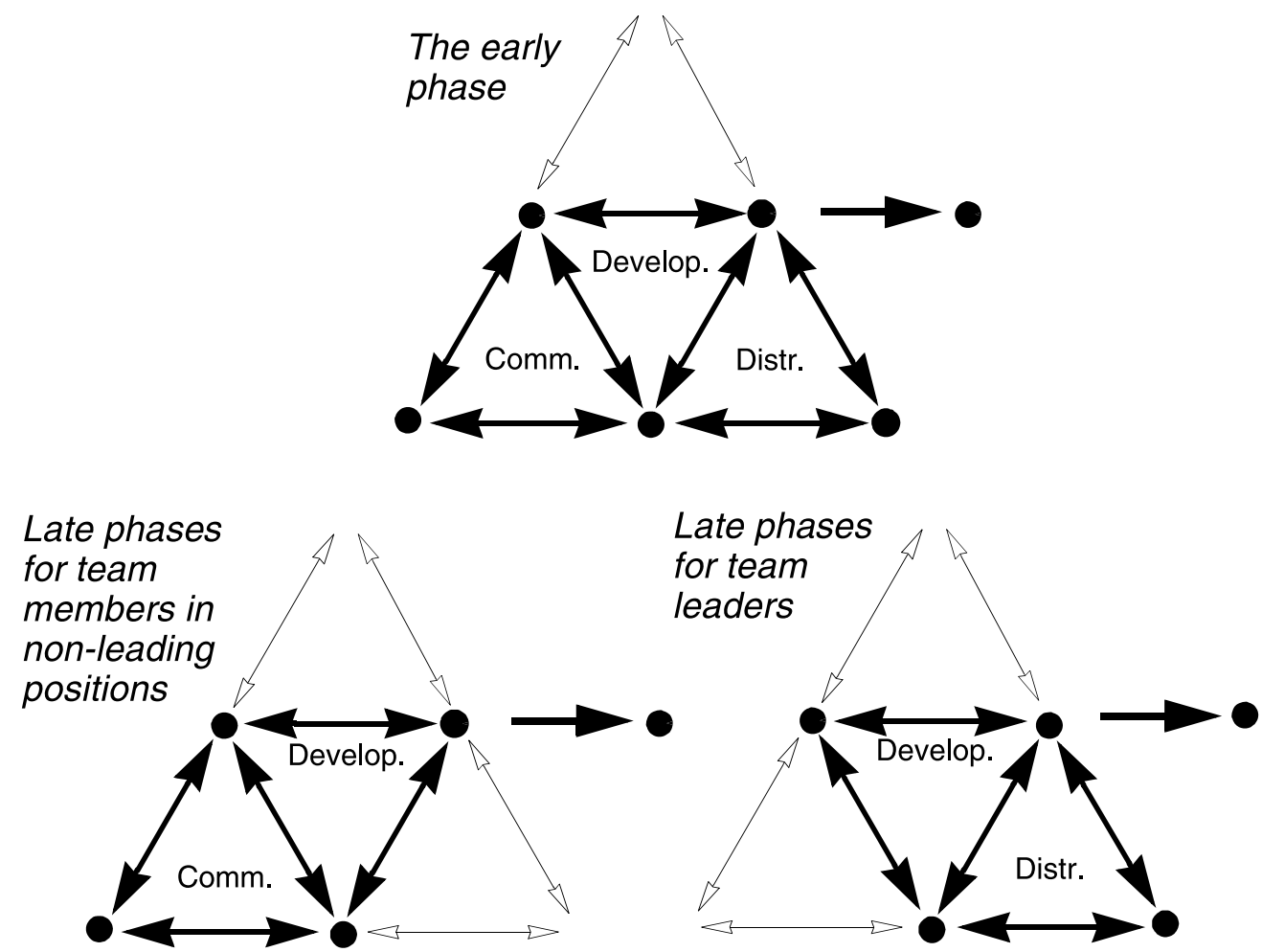

Figure 4. The development of the experienced control structure over time for the phenomenographic category Control is allocated. The top sketch indicate the situation as experienced in the early phase of the group, the down left sketch depicts how the situation is experienced for a team member in a non-leading position, while the down right shows the situation as experienced by the leader 
This category describes a change. Initially, as a new team is constituted, it selects a leader, to whom the right to take and implement decisions is allocated. The rules for the collaboration as well as the division of control and labour are negotiated. At later stages, when the rules are set, the situation develops so that the leader comes to act on behalf on his/her team taking decisions and distributing tasks. The roles of members in non-leading positions also develop to become dominated by the rules. The sketches in Figure 4 represent this change. After the initial phase (upper triangle), the leader experiences a situation where distribution and development form the core, as illustrated in the lower left sketch. For a team member in a non-leading function communication and development dominate in their experience of the situation instead (lower right sketch).

In general, this category represents a control structure that functions well. Problems are only rarely reported by team members who do not have a leadership position. One of the team leaders, has, on the other hand, vented their frustration, expressed in a conflict between his duties as a team leader to distribute tasks and the absence of meaningful ways to enforce his leadership.

Some aspects of the activity theoretical framework can shed further light on the problem he expresses. When the team is working with the code, after the initial decisions the rules about how the work should be performed are applied. If a problem arises, it cannot easily be solved through a redefinition of rules in the on-going process, since rule-constructing activities have now ceased (as illustrated in the lower right triangle) and the leader no longer has the capacity to influence the rules. The process of redefinition, discussed above as an aspect of the first phenomenographic category, is not directly available at later stages. To overcome this contradiction, a developmental change of the system itself is needed (Engeström, 1987), that is, new decisions about the control structure need to be taken within the team. Comparing this with the first category, in which rules can continuously be renegotiated, a critical difference between the two categories has thus been discerned. These results are well in line with the findings of Hause (2003), who from a study drawing its data from the interaction between the team members in the same course, through the application of discourse analysis, has shown that decentralized groups show high quality outcomes.

\section{Discussion: what can be learned from the case study?}

This case study illustrates how the framework can be deployed to enlighten findings from a phenomenographic analysis through the use of elements from activity theory. The development over time, and the possible conflicts that lie hidden in the different ways that something (here the control structure) is experienced, are brought to light. In this example, a contradiction hidden within one of the phenomenographic categories has become visible, and a control structure where the control is distributed has been identified as more desirable than one where the control is allocated to the team leader.

In this context, it must be remembered that the categories are idealised descriptions that are created by the researcher. This implies that the categories do not correspond 
to specific 'real' teams. Instead, they serve as patterns that help the researcher in his/ her task of analysing the learning situation. Thus one cannot draw the conclusion that a team, which by participants is described as having a distributed control structure, is automatically a well-functioning team. Turning the statement around is possible, however: a distributed control structure is one (of the) desirable control structure(s) and thus ought to be encouraged through course design.

Of course, the case study presented here does not represent a complete activity and does not give justice to the complexity and richness of activity theory. The final analyses of this project will encompass not only one issue, such as control, but also other phenomena studied in the phenomenographic tradition. The activity will then be taken as a whole and will describe a system where the activity models the students' experience of their learning situation. However, already a small example of this kind, where only some ways of experiencing one phenomenon have been scrutinised, illustrates how the framework can serve as a vehicle for the researcher to learn about the students' experiences and thus can inform course design.

\section{Conclusions}

The framework proposed in this paper is intended to allow the study of learning concepts within a subject area in a complex learning environment. It offers ways of analysing and interpreting the learning that takes place in distributed project-based courses, and enables identification of critical factors in the learning environment that promote-or hinder-learning. The framework is based upon phenomenography, extend by concepts from activity theory. In this way the perspective of the learner, and the importance of the subject area (both key features of phenomenography) are kept but the scope is enriched from focusing mainly on the variation in experience of particular phenomena to encompassing the whole learning environment in the different ways that learners experience it. As experienced contradictions and conflicts become visible for the researcher, insights into how students go about collaborating are gained, and the different elements in the course environment can be related to collaboration and learning.

Through use of the framework, an analysis of the variation in the experience of a learning environment as a whole is enabled. In this respect it offers richer results for studies concerning the learning environment than phenomenography alone, in which issues contextual to the phenomenon under investigation are relegated to the background. Since students experience their learning environment as a whole- and not as a set of phenomena - this feature of the framework is valuable for the study of complex learning environments.

In contrast to studies performed in the activity theoretical tradition per se, the students' experience is the keystone of the proposed framework. To take the students' ways of experiencing the learning environment into account offers ways of designing well-functioning courses. Changes in course design must be based on the learners' experiences if they are not to be counter-productive to the learning aims. This aspect is highlighted through an example in the article, where one way of experiencing the 
structure of control in a virtual team is recognised to be more fruitful than another, with regard to how well the group functions as a unit. Course designers should consider such issues if they are to develop courses that promote desirable structures of collaboration within teams.

Through its focus on the students' ways of experiencing learning in an experienced whole, the framework contributes to research and development in computersupported distributed project-based courses that promote students' learning of the subject area.

\section{Acknowledgements}

This paper is based on a research paper presented at ALT-C 2003 and published in the conference's research proceedings.

This project is financed by The Knowledge Foundation of Sweden and the Swedish Research Council. Shirley Booth and Arnold Pears have contributed with valuable points of view during the course of this research project.

\section{References}

Åberg-Bengtsson, L. (1998) Entering a graphicate society: young children learning graphs and charts (Gothenburg, Sweden, Acta Universitatis Gothoburgensis).

Adawi, T., Berglund, A., Booth, S. \& Ingerman, Å (2001) On context in phenomenographic research on understanding heat and temperature, in: A. Berglund. On the understanding of computer network protocols, Licentiate thesis, Department of Information Technology, Uppsala University. Available online: http://www.docs.uu.se/ andersb/lic/ (accessed 25 November 2003).

Bannon, L. (1997) Activity theory. Available online: http://www-sv.cict.fr/cotcos/pjs/TheoreticalApproaches/Actvity/ActivitypaperBannon.htm (accessed 25 November 2003).

Bellamy, R. K. E. (1996) Designing educational technology: computer-mediated change, in: B. Nardi (Ed.) Context and consiousness, activity theory and human-computer interaction (Cambridge, MA, MIT Press).

Berglund, A. (2002a) On the understanding of computer network protocols, Licentiate thesis, Department of Information Technology, Uppsala University. Available online: http:// www.docs.uu.se/ andersb/lic/ (accessed 25 November 2003).

Berglund, A. (2002b) Learning computer systems in a distributed course: problematizing content and context, paper presented at the European Association for Research into Learning and Instruction, SIG 10, Current Issues in Phenomenography, Canberra, Australia.

Berglund, A. (in press) Learning computer networks in a distributed learning environment, Unpublished PhD thesis, Department of Information Technology, Uppsala University.

Booth, S. (1992) Learning to program: a phenomenographic perspective (Gothenburg, Sweden, Acta Universitatis Gothoburgensis).

Cope, C. (2000) Educationally critical aspects of the experience of learning about the concept of an information system, unpublished $\mathrm{PhD}$ thesis, La Trobe University.

Coupland, M. \& Crawford, K. (2002) Researching complex systems of activity, paper presented at the European Association for Research into Learning and Instruction, SIG 10, Current Issues in Phenomenography, Canberra, Australia. Available online: http://www.anu.edu.au/cedam/ ilearn/symposium/Coupland.doc.

Daniels, M. (1999) Runestone, an international student collaboration project, NyIng report No 11, Linköping. 
Ekeblad, E. \& Bond, C. (1994) The nature of a conception: questions of context, in: R. Ballantyne \& C. Bruce (Eds.) Phenomenography: philosophy and practice (Brisbane, Queensland University of Technology).

Engeström, Y. (1987) Learning by expanding: an activity-theoretical approach to developmental research (Helsinki, Orienta-konsultit).

Engeström, Y. (1993) Developmental studies of work as a test of activity theory: the case of primary care medical practice, in: S. Chaiklin \& J. Lave (Eds) Understanding practice-perspectives on activity and context (Cambridge, Cambridge University Press), 64-103.

Gordon, S. (1998) Understanding students learning statistics: an activity theory approach, unpublished doctoral dissertation, The University of Sydney. Available online: http://adt.caul.edu.au (accessed 25 November 2003).

Hause, M. L. (2003), Software development performance in remote student teams in international computer science collaboration, unpublished $\mathrm{PhD}$ thesis, The Open University.

Hultén, M. \& Booth, S. (2002) Considering context for networked learning in a phenomenographic perspective, paper presented at Networked Leaning 2002, Sheffield, UK.

Kaptelinin, V., Kuutti, K. \& Bannon, L. (1995) Activity theory: basic concepts and applications, in: B. Blummenthal, J. Gornostaev \& C. Unger (Eds.) Lecture notes in computer science, 1015 (Berlin, Springer), 188-201.

Kuutti, K. (1996) Activity theory as a potential framework for human-computer interaction research, in: B. Nardi (Ed.) Context and consiousness, activity theory and human-computer interaction (Cambridge, MA, MIT Press), 17-44.

Marton, F. \& Booth, S. (1997) Learning and awareness (Mahwah, NJ, Lawrence Erlbaum Associates).

Nardi, B. (1996) Activity theory and human-computer interaction, in: B. Nardi (Ed.) Context and consiousness, activity theory and human-computer interaction (Cambridge, MA, MIT Press).

Pang, M. F. (2003) Two faces of variation: on the continuity in the phenomenographic movement, Scandinavian Fournal of Educational Research, 47(2), 145-156. 